\title{
Sistema para el monitoreo remoto de la temperatura en transformadores de distribución
}

\author{
Remote Monitoring System For The Temperature Distribution Transformers \\ Juan Sebastián Mayuza López, Iván Mariscal Lozano, Edwin Andrés Quintero Salazar \\ Ingeniería Electrónica, Grupo de Investigación en Astronomía Alfa Orión, Universidad Tecnológica de \\ Colombia, Pereira, Colombia \\ Correo-e: equintero@utp.edu.co
}

\begin{abstract}
Resumen- En este documento se presenta el desarrollo de un prototipo para el monitoreo remoto de la temperatura presente en transformadores de distribución. El sistema opera con dos señales de entrada, una proveniente de la temperatura existente en la parte interna del transformador, y otra correspondiente a la medición del valor ambiente. Posteriormente, las señales pasan a una etapa de acondicionamiento por medio de amplificadores operacionales, para finalmente ser transmitidas mediante módulos de radiofrecuencia. En el receptor, se realiza la visualización de los valores correspondientes en una pantalla de cristal líquido. El sistema se instaló en uno de los transformadores de distribución del laboratorio de máquinas eléctricas de la Universidad Tecnológica de Pereira, realizando 20 mediciones de temperatura con el sistema de medición y con el equipo patrón, a diferentes horas del día y con diferentes cargabilidades para el transformador, obteniéndose un error máximo del 3,09\%.
\end{abstract}

Palabras clave- Acondicionamiento de señal, módulo de radiofrecuencia, sensor de temperatura, transformador de distribución.

\begin{abstract}
This paper presents the development of a prototype for remote monitoring of temperature, present in distribution transformers. The system operates with two input signals, one from the temperature at the inside of the transformer, and another for measuring the ambient value. Thereafter, the signals pass to a conditioning stage by means of operational amplifiers, to finally be transmitted via radio frequency modules. At the receiver, the corresponding values are displayed on a liquid crystal display. The system was installed in one of the distribution transformers, existing in the electrical machines laboratory at the Universidad Tecnológica de Pereira. 20 measurements were performed using the temperature measurement system, and the standard instrument, at different times of day and different cargabilidades for transformer, yielding a maximum error of $3.09 \%$.
\end{abstract}

Key Word - Signal conditioning, radio frequency module, temperature sensor, distribution transformer.

\section{INTRODUCCIÓN}

Fecha de Recepción: 28 de Enero de 2015

Fecha de Aceptación: 14 de Diciembre de 2015
Los transformadores eléctricos son máquinas estáticas que se encargan de transformar el nivel de tensión de corriente alterna que se encuentra en la entrada de este dispositivo, en otro valor diferente a la salida del mismo, mediante la acción de un campo magnético [1]. Este principio de funcionamiento es aplicado a cualquier nivel de tensión que se maneje en los sistemas eléctricos de potencia. Existen algunos factores que influyen en el buen desempeño de estos equipos, y que, debido a su gran importancia para la vida diaria, es necesario mantener controlados, pues es debido a estos que se pueden presentar fallas. Uno de estos aspectos críticos es la temperatura. Existen varias causas fundamentales por las cuales se puede presentar el calentamiento de los transformadores en condiciones anormales. Una de ellas es la sobrecorriente, la cual se encuentra presente durante el arranque de motores eléctricos, debido a las elevadas corrientes que son superiores a las nominales durante un corto periodo de tiempo, pero que al realizarse en forma repetitiva, sí el transformador es sobredimensionado, puede verse seriamente sobrecargado [2]. De igual forma, un bloqueo de algún motor por un significativo periodo de tiempo puede provocar una sobrecarga prolongada.

Por otra parte, las cargas inductivas, los rectificadores, y la gran mayoría de los convertidores, introducen armónicos y tasas de distorsión que puede ser bastante elevados, lo cual se manifiesta mediante picos de tensión que suponen una desclasificación de la potencia del transformador, generándose de esta manera un incremento de la temperatura. De igual forma, los cortocircuitos y las frecuentes puestas en conexión, generan calentamientos con cierta intensidad [3]. Así mismo, la sobretensión provocada por sobreinducción, que puede originarse en la mala regulación de la red eléctrica, y las pérdidas generadas por el circuito magnético del transformador; implican un sobre calentamiento de todo el equipo [4]. Otra fuente de sobreinducción consiste en los valores inferiores de frecuencia presentes en la red, para los cuales naturalmente no ha sido diseñado el transformador. Otro factor que influye en el incremento de la temperatura en estos equipos, consiste en el envejecimiento del aceite, ya que cada vez que el transformador se sobrecarga con relación a su funcionamiento nominal, existe sobrecalentamiento, y por lo 
tanto reducción en su ciclo de vida [5]. Con el objetivo de mantener controlada esta variable para así garantizar el correcto funcionamiento del transformador, es de vital importancia implementar un sistema de instrumentación confiable, capaz de proporcionar la cantidad de datos suficiente como para proceder a ejecutar operaciones de control. Actualmente, existen dispositivos como el DTM CA027 de la empresa Brasileña TreeTech®, el cual utiliza cuatro sensores PT100 con el fin de establecer la temperatura de diferentes secciones del transformador. Este dispositivo posee un puerto de comunicación serial por lazos de corriente $R S 485$, mediante el cual es posible exportar la información obtenida [6]. Otro ejemplo claro de la implementación de sensores PT100 en este tipo de aplicaciones, consiste en el sistema ideado por J. Q. Feng, P. Sun, W. H. Tang, D. P. Buse, Q. H. Wu, Z. Richardson, y J. Fitch; presentado en la Conferencia Internacional de Tecnología de Sistemas de Potencia, realizada en el año 2002. En este trabajo se presenta un sistema de monitoreo de temperatura basado en sensores $N T C$, el cual involucra un sistema de almacenamiento de información, y una interfaz que posibilita la comunicación vía protocolo HTTP, con el objetivo de montar los datos a un servidor que posibilite la visualización de los mismos vía internet [7]. Por su parte, Shiyong Pan, Ping Zheng, Yinlu Ren, Changju Chen, y Zihui Shao; idearon un sistema de monitoreo de temperatura que permite involucrar los sensores de toda la planta o subestación, dentro de una red de fibra óptica, lo cual posibilita el monitoreo remoto de todo el sistema, además de facilitar el reemplazo de los elementos defectuosos, toda vez que los sensores se convierten en equipos de red de fácil sustitución [8]. Con respecto a las aplicaciones robustas existentes hoy en día, que buscan sostener un monitoreo permanente de todas las variables involucradas en una subestación de energía eléctrica, David Dolezilek y Brian McDermott, realizaron una presentación en la $18^{\mathrm{a}}$ Conferencia Internacional de Distribución de Electricidad, en la cual describen los dispositivos electrónicos inteligentes (IED) involucrados en los sistemas de control, supervisión y adquisición de datos, más conocidos con la sigla $S C A D A$. El equipo al cual dedican su trabajo, es el Legacy $P \& C$, basado en relés electromecánicos y sistemas de telemetría SCADA. Esta solución permite el monitoreo de tensión, corriente, y temperatura en los devanados y en el ambiente en el cual se encuentra inmerso en transformador [9].

Sin embargo, las soluciones descritas anteriormente, están basadas en redes cableadas, ya sea eléctricas o de fibra óptica, buscando siempre la inclusión del sistema de medición en redes de información. En cuanto a lo que tiene que ver con el monitoreo inalámbrico, se encuentran muy pocos desarrollos, dentro de los cuales se destaca el ideado por Wu Chunming y Geng Qiang, consistente en una estación remota de medición de temperatura en transformadores que transmite los datos a una estación base mediante la red GPRS de la empresa China Mobile Telecommunication Company, para finalmente ser montados en un servidor Web que permite el acceso a usuarios autorizados. El sistema está basado en el microcontrolador $S 3 C 44 B 0 X$ de la firma Samsung ${ }^{\circledR}$, el cual maneja todo el protocolo de comunicación [10]. Analizando el tema desde el punto de vista nacional, aun no existen redes de datos extensas y confiables que interconecten los transformadores de distribución, por lo cual en este trabajo se propone la implementación de un medidor que permita al operario de la empresa de energía, monitorear la temperatura presente en el transformador de manera inalámbrica, sin tener contacto directo con el equipo. De esta forma se reducen los riesgos derivados de la interacción del técnico con la máquina.

Así pues, a continuación se describe la ejecución de un proyecto de investigación en el cual se propone el diseño y la construcción de un prototipo para el monitoreo remoto de la temperatura presente en los transformadores de distribución, basado en radiofrecuencia; el cual involucra el uso de sensores semiconductores de temperatura, microcontroladores, acondicionadores de señal, módulos de comunicación inalámbrica, y visualizadores de cristal líquido.

\section{DESARROLLO DEL PROTOTIPO}

\section{A. Estructura General del Sistema}

El prototipo desarrollado se encuentra conformado por varias etapas, siendo cada una de ellas fundamentales para lograr el buen desempeño del mismo. Inicialmente, es necesario conocer el tipo de sensor que se desea utilizar, con el objetivo de abarcar el rango de temperatura presente en los transformadores de distribución. Acto seguido, se debe identificar el procedimiento mediante el cual se realiza el acondicionamiento de la señal, para así posteriormente establecer la mejor técnica a utilizar para la comunicación de la información de manera inalámbrica; finalizando con la visualización de los datos obtenidos en el receptor. En la Figura 1 es posible apreciar el diagrama de bloques del sistema desarrollado.

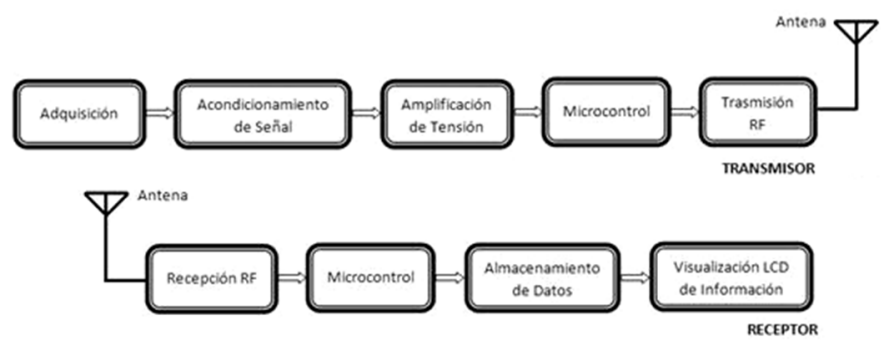

Figura 1. Diagrama de bloques del prototipo desarrollado.

\section{B. Medición de la Temperatura}

Con el objetivo de obtener el valor de la temperatura presente en los transformadores de distribución, es necesario hacer uso de un dispositivo electrónico conocido como transductor. Este elemento permite convertir la magnitud física que se va a 
medir en una señal de salida (normalmente tensión o corriente) que puede ser procesada por el sistema de acondicionamiento, a menos que la señal a detectar sea eléctrica. En forma general, el transductor es un elemento que transforma energía. Según lo anterior, el transductor debe tomar poca energía del sistema bajo observación, para no alterar la medición [11]. El sensor de temperatura utilizado en el desarrollo del presente proyecto fue el dispositivo semiconductor LM35, introducido en 1983 por National Semiconductors Inc. [12], elegido gracias a que ofrece un alto rango de medición a un bajo precio, lo cual permite convertir al prototipo en un sistema eficiente y viable desde el punto de vista económico. El LM35 tiene una salida de $10 \mathrm{mV} /{ }^{\circ} \mathrm{C}$, con una no-linealidad típica de $\pm 1 / 4{ }^{\circ} C$, sobre un rango de temperatura que va desde -55 hasta $+150^{\circ} \mathrm{C}$. La eficiencia de este dispositivo se encuentra asegurada por su bajo costo, y facilidad de ajuste y calibración. La baja impedancia en la salida del LM35, su salida cuasi-lineal, y la calibración inherente de precisión, hacen de la toma de datos una tarea sencilla. Puede ser usado con una sola fuente de alimentación, o con las secciones positiva y negativa de la fuente. Como solo utiliza $60 \mu \mathrm{A}$ de la fuente de alimentación, presenta un calentamiento muy bajo (menos de $0,1^{\circ} \mathrm{C}$ al aire libre). La serie $L M 35$ es empaquetada en el transistor hermético TO-46, mientras que el $L M 35 C$, el LM35CA y el $L M 35 D$ se encuentran en un paquete de transistor plástico tipo TO-92. La Figura 2 presenta un esquema típico para la conexión del dispositivo anteriormente mencionado.

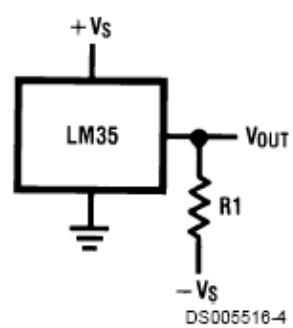

$$
\begin{aligned}
& \text { Choose } R_{1}=-V_{S} / 50 \mu \mathrm{A} \\
& \begin{aligned}
V_{\text {OUT }} & =+1,500 \mathrm{mV} \text { at }+150^{\circ} \mathrm{C} \\
& =+250 \mathrm{mV} \text { at }+25^{\circ} \mathrm{C} \\
& =-550 \mathrm{mV} \text { at }-55^{\circ} \mathrm{C}
\end{aligned}
\end{aligned}
$$

Figura 2. Esquema para la conexión y operación del sensor de temperatura $L M 35$.

\section{Acondicionamiento de Señal}

El circuito de acondicionamiento se encuentra conformado por varias etapas. Inicialmente, se introduce la señal de tensión arrojada por el sensor a un seguidor de tensión, utilizado con el fin de eliminar los ruidos indeseados introducidos en el proceso de medición. Además, esto permite realizar un acople de impedancias de tal manera que no se afecte la medición al conectar más circuitos al sistema [13]. Posteriormente, se encuentra un amplificador operacional en configuración no inversora, utilizado para elevar el nivel de tensión suministrado por el seguidor. El proceso finaliza con un conversor análogo digital involucrado dentro del microcontrolador PIC16F876A, necesario para enviar la información a transmitir de manera digital al módulo transmisor de $R F$.

\section{Microcontrolador}

Los microcontroladores son computadores digitales integrados en un chip que cuentan con un microprocesador o unidad de procesamiento central (CPU), una memoria para almacenar el programa, una memoria para almacenar datos y puertos de entrada salida. A diferencia de los microprocesadores de propósito general, como los que se usan en los computadores PC, los microcontroladores son unidades autosuficientes y más económicas. El funcionamiento de los microcontroladores está determinado por el programa almacenado en su memoria. Este puede escribirse en distintos lenguajes de programación. Además, la inmensa mayoría de los microcontroladores actuales pueden reprogramarse repetidas veces [14]. Para el presente proyecto se utilizó el microncontrolador PIC16F876A, fabricado por Microchip. Este dispositivo es el más popular para aplicaciones de nivel intermedio, y es una excelente opción cuando se requieren más líneas de entrada/salida, convertidores $A / D$, señales $P W M$, comunicación serial por hardware, entre otros aspectos. Existen versiones con distintas características, tales como el tamaño de la memoria y número de terminales. Se decidió utilizar este elemento ya que permite realizar la conversión de la señal análoga suministrada por el circuito de acondicionamiento, en una señal digital, apta para ser enviada a los módulos de comunicación $R F$. En la Figura 3 es posible apreciar la distribución de terminales del PIC16F876A.

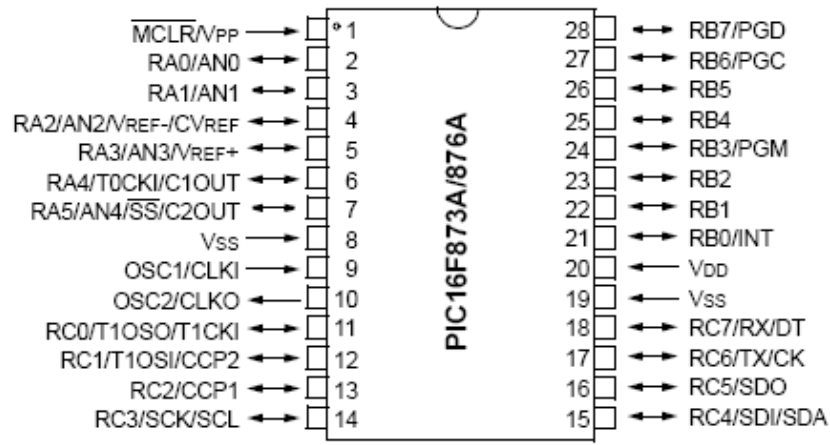

Figura 3. Distribución de pines del microcontrolador PIC16F876A.

\section{E. Módulos de Comunicación por Radio Frecuencia}

Los módulos $R F$ pueden utilizar distintos tipos de modulación y técnicas de radio. Entre las técnicas de modulación que pueden emplear se encuentran:

- Modulación por desplazamiento de amplitud.

- Modulación por desplazamiento de frecuencia.

- Modulación por desplazamiento de fase.

- Modulación por desplazamiento de fase múltiple.

- Modulación de amplitud en cuadratura. 
En cuanto a inmunidad al ruido y cobertura se refiere, la modulación por desplazamiento de fase binaria $(B P S K)$ ofrece gran confiabilidad con amplios rangos de cobertura, aunque su montaje es un poco complejo y requiere de una buena inversión de dinero. Como la solución planteada en este trabajo pretende facilitar el acceso del técnico electricista a los datos provenientes del transformador ubicado a escasos metros sobre el suelo, no se requieren sistemas de comunicación muy exigentes ni con coberturas demasiado extensas. Dado lo anterior, se optó por utilizar módulos de $R F$ basados en la técnica de modulación por desplazamiento de amplitud $(A S K)$, la cual ofrece una cobertura moderada sin incurrir en altos costos.

Para el desarrollo del proyecto se utilizaron los módulos de comunicación $R F$ referencia $F S 1000 A$, los cuales utilizan la modulación por desplazamiento de amplitud $(A S K)$, para emitir una señal de radio frecuencia de $20 \mathrm{~mW}$ de potencia. En la Tabla 1 es posible apreciar las principales características de los módulos utilizados, mientras que en la Figura 4 se presenta el diagrama de conexión y la distribución de terminales.

\begin{tabular}{|l|l|}
\hline Voltaje de Operación & $3-12 \mathrm{VDC}$ \\
\hline Corriente de Operación & $5-45 \mathrm{~mA}$ \\
\hline Tasa Máxima de Transferencia & $2.4-9.6 \mathrm{kHz}$ \\
\hline Modulación & ASK \\
\hline Frecuencia de Operación & $433 \mathrm{MHz}$ \\
\hline Alcance & $15 \mathrm{~m}$ \\
\hline Potencia de Emisión & $20 \mathrm{~mW}$ \\
\hline Dimensiones & $19 \mathrm{~mm} \times 19 \mathrm{~mm} \mathrm{x}$ \\
& $8 \mathrm{~mm}$ \\
\hline
\end{tabular}

Tabla 1. Principales características de los módulos RF FS1000A.

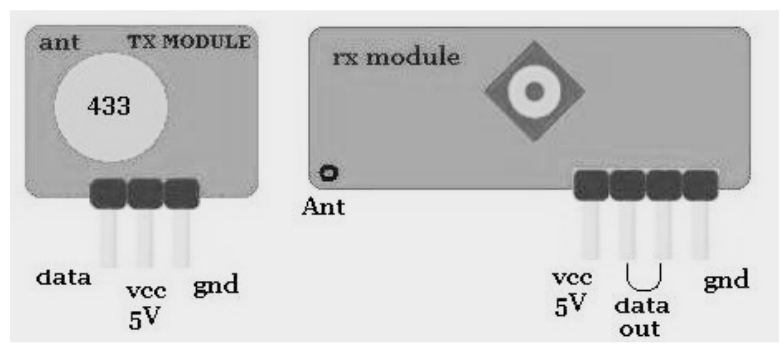

Figura 4. Distribución de terminales de los módulos RF FS1000A.

Con el fin de ofrecer una cobertura de hasta $15 \mathrm{~m}$, las inductancias de los módulos transmisor y receptor actúan como antenas. Dado que este proyecto contempla el establecimiento de una comunicación inalámbrica corta entre el operario de la empresa de energía y el transformador en observación, en el sitio en el cual este último se encuentra instalado, no se hace necesaria la implementación de antenas externas. Sin embargo, el fabricante de los módulos ofrece varias versiones de este elemento mediante los cuales es posible incrementar la cobertura de la comunicación.
Inicialmente, se procedió a adquirir un nivel de tensión equivalente a la temperatura detectada por el componente electrónico LM35 descrito en apartados anteriores, el cual tiene un comportamiento cuasi lineal, que relaciona la temperatura con el valor de tensión obtenido. Esta señal es ingresada al sistema de acondicionamiento de señal, en el cual se acopla la impedancia y se asigna cierta ganancia de voltaje. En la Figura 5 se presenta el diagrama esquemático final diseñado para esta etapa.

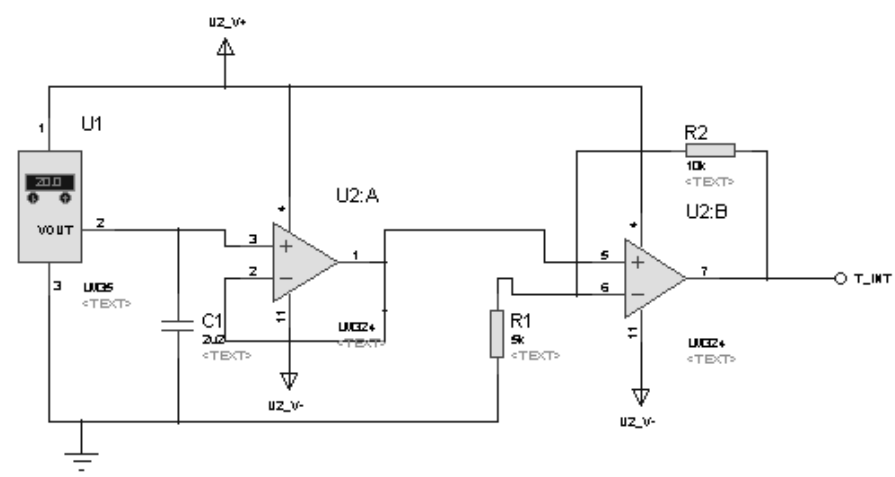

Figura 5. Diagrama esquemático del circuito de detección, acondicionamiento y amplificación de señal diseñado para el prototipo.

Este circuito está conformado por tres etapas básicas:

- Sensor de temperatura LM35 (adquisición).

- Seguidor de tensión (acondicionamiento).

- Amplificador operacional no inversor (amplificación de tensión).

Este procedimiento se realiza tanto para adquirir la temperatura interna del transformador, como para la temperatura externa, es decir, la temperatura ambiente. Cabe aclarar que ambos circuitos son completamente independientes.

Al obtener ambas temperaturas, cuya representación es completamente analógica, las señales son ingresadas a los terminales 2 y 3 del microcontrolador, para realizar la conversión análoga digital, y así de esta forma realizar el procesamiento correspondiente. Después de adquirir ambas señales, se procede a alistarlas dentro del microcontrolador PIC16F876A involucrado en el transmisor, para enviarlas a través del módulo $R F$. El programa grabado en el microcontrolador transmisor se puede resumir en el diagrama de flujo presentado en la Figura 6 (sección izquierda). Para iniciar la comunicación, el microcontrolador entrega los datos mediante protocolo serial al módulo transmisor $R F$. En la Figura 7 es posible apreciar la forma de onda de los datos entregados por el microcontrolador ubicado en el transmisor.

\section{F. Construcción del Prototipo}




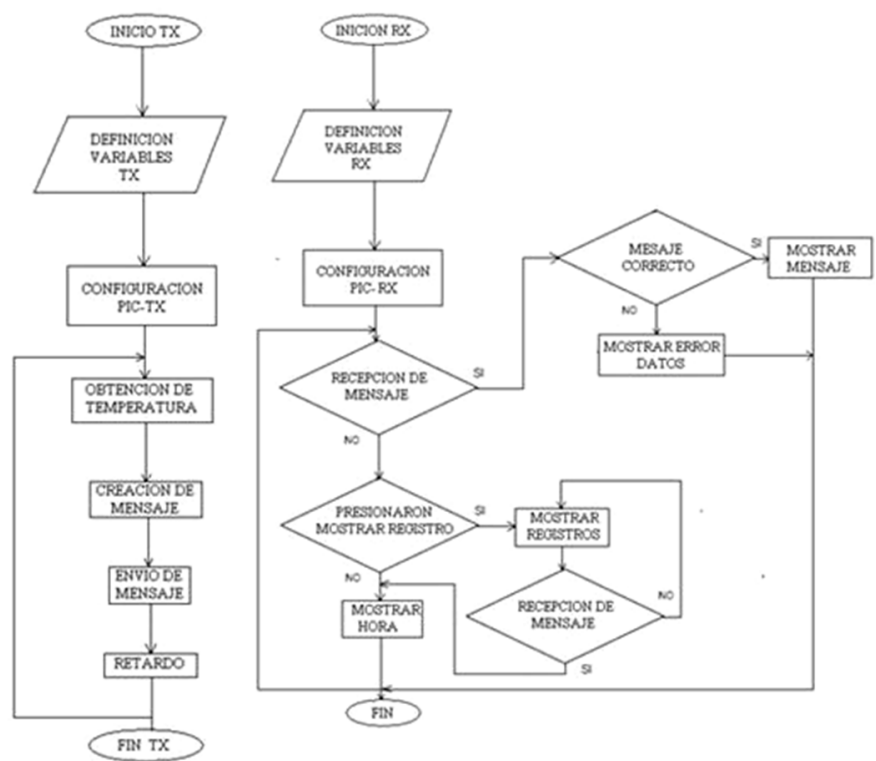

Figura 6. Diagrama de flujo del programa almacenado en el microcontrolador.

Una vez establecida la comunicación, el módulo receptor de $R F$ arroja una señal digital, la cual es acondicionada mediante un comparador de tensión, para ser ingresada finalmente al microcontrolador receptor, el cual se encarga de entregar los datos a una pantalla de cristal líquido $(L C D)$. En la Figura 6 (sección derecha) es posible apreciar el diagrama de flujo del programa almacenado en el microcontrolador PIC16F876A utilizado en el equipo receptor, mientras que la Figura 8 hace lo propio para las subrutinas de interrupciones.

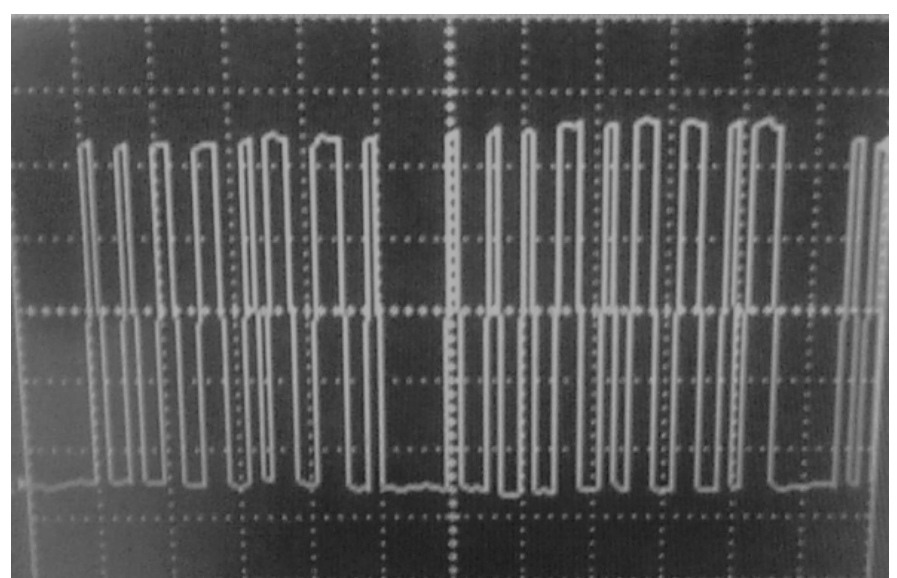

Figura 7. Señal a transmitir por el módulo RF.

\section{G. Prototipo Final}

En las Figuras 9 y 10 es posible apreciar los diagramas esquemáticos finales diseñados para el transmisor y para el receptor respectivamente.

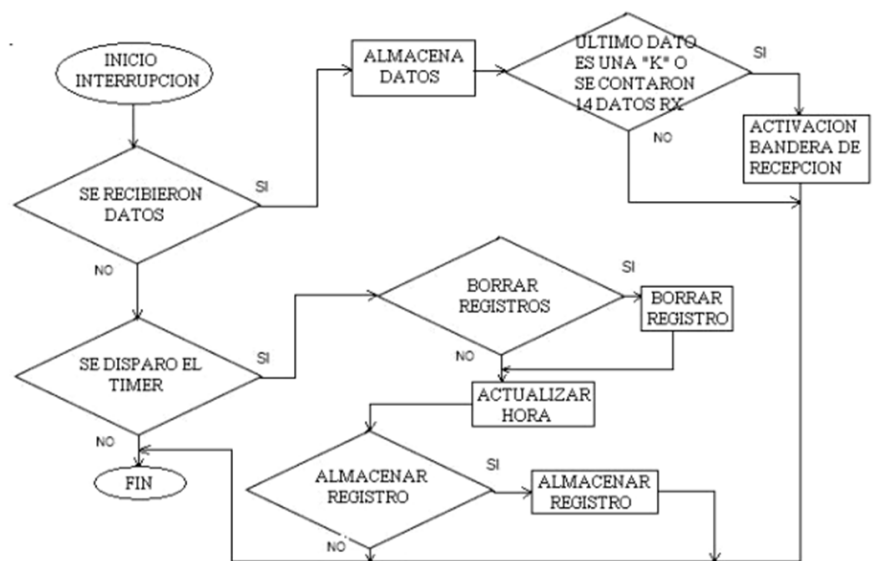

Figura 8. Diagrama de flujo para las subrutinas de interrupciones.

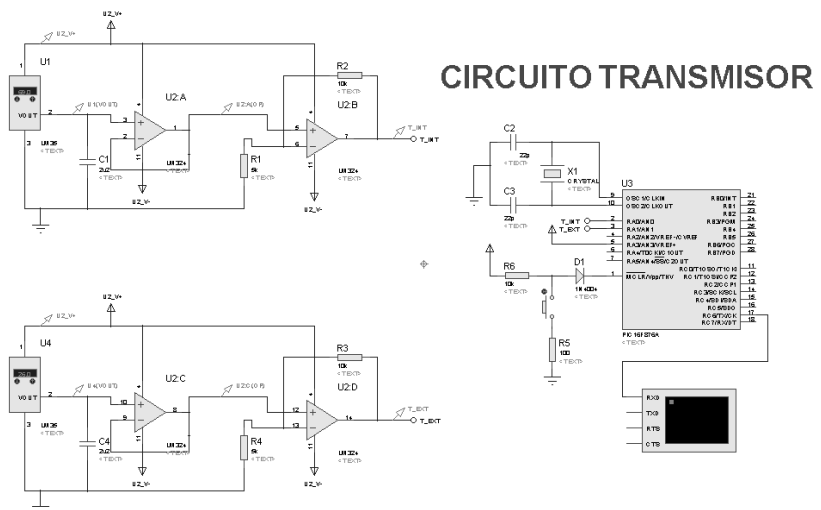

Figura 9. Diagrama esquemático del transmisor.
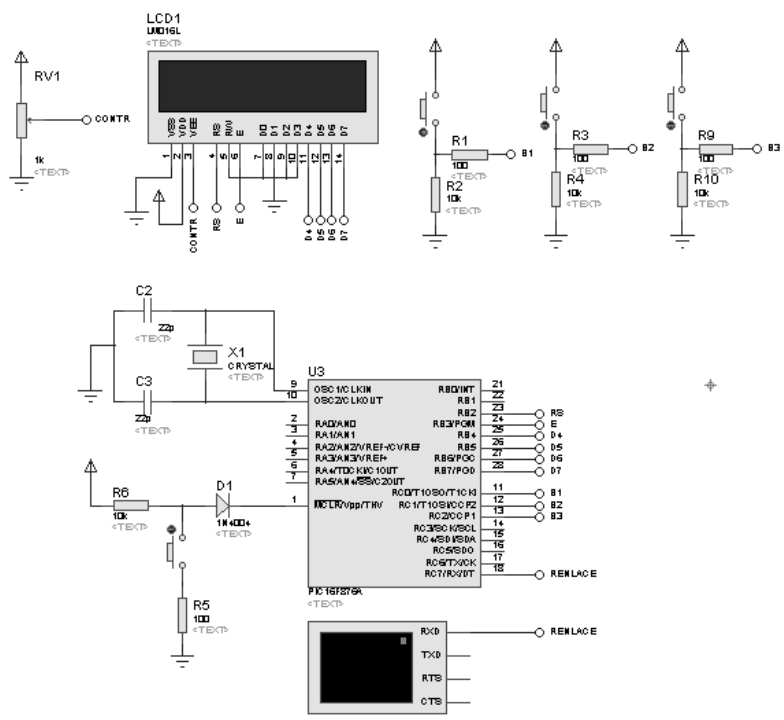

Figura 10. Diagrama esquemático del receptor.

Las Figuras 11 y 12 contienen fotografías tomadas a los sistemas de transmisión y recepción diseñados en el proyecto de medición remota de la temperatura presente en los transformadores de distribución. 


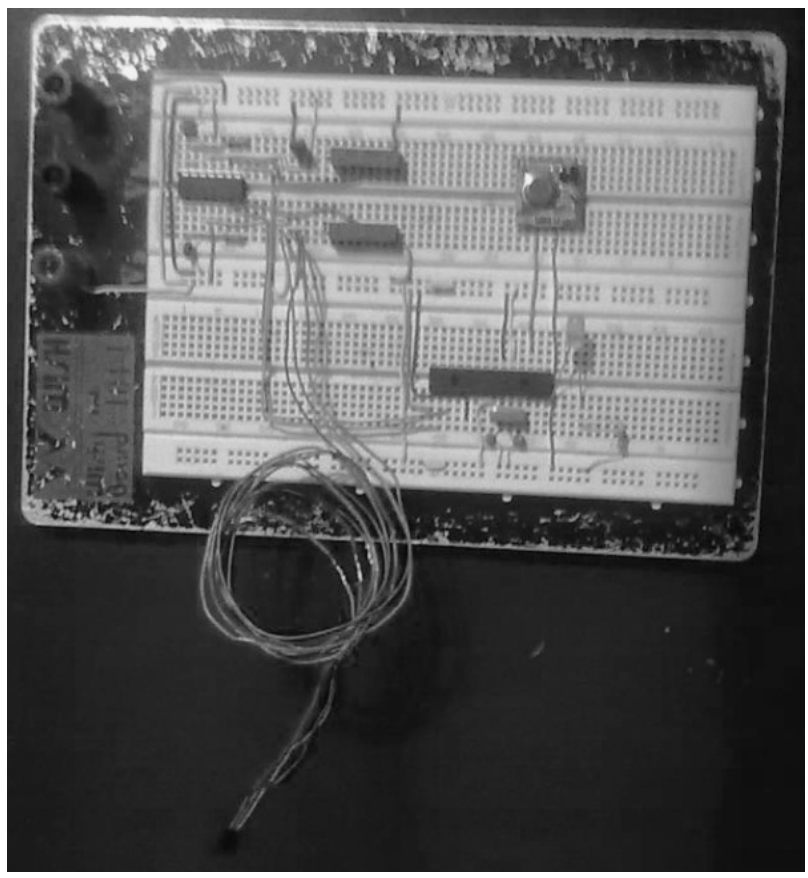

Figura 11. Aspecto físico del módulo transmisor, perteneciente al prototipo desarrollado.

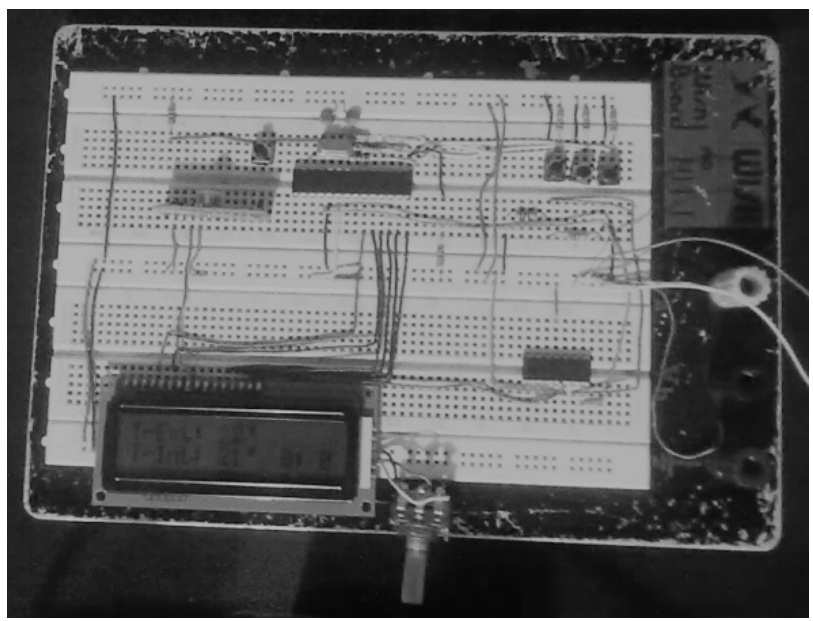

Figura 12. Fotografía del módulo receptor construido para el prototipo de medición de la temperatura de los transformadores de distribución.

\section{RESULTADOS}

A continuación se presentan los datos obtenidos al realizar la medición de la temperatura interna en uno de los transformadores de distribución del laboratorio de máquinas eléctricas de la Universidad Tecnológica de Pereira. Los valores arrojados por el prototipo construido fueron cotejados con los suministrados por el termómetro del multímetro digital $P D-751$ de la marca Fluke. También se muestran las gráficas correspondientes, en las cuales es posible observar el comportamiento cuasi lineal de sensor LM35 y el funcionamiento del sistema de acondicionamiento de señal. En la Tabla 2 se presentan los datos obtenidos para la medición de la temperatura interna del transformador y en el medio ambiente. Allí es posible apreciar los valores arrojados Ppor el multímetro y los suministrados por el prototipo. Al analizar los datos obtenidos, se observa que el error porcentual más alto fue de $3,3 \%$ para la temperatura interna del transformador, y de 5\% para la temperatura ambiente. Para la realización de la prueba, se utilizó un transformador de distribución de $150 \mathrm{kVA}$, trifásico, conectado a media carga de manera balanceada. Cada 5 minutos se adelantaron modificaciones a la carga conectada y se cambiaron las condiciones del entorno, con el objetivo de generar variaciones en la temperatura interna del transformador. Los datos de la Tabla 2 corresponden a 20 mediciones de temperatura, tomadas a intervalos de 5 minutos, para un total de 1:40 horas de prueba; desarrolladas el 23 de mayo de 2012 de 14:00 a 15:40H. Los sensores de temperatura del equipo Fluke $\odot$ de referencia, y del prototipo desarrollado, fueron instalados en uno de los bobinados expuestos de la máquina.

\begin{tabular}{|c|c|c|c|c|c|}
\hline $\begin{array}{c}\text { Temperatura } \\
\text { Interna Medida } \\
\text { por el } \\
\text { Termometro } \\
\text { Fluke }\left({ }^{\circ} \mathrm{C}\right) \\
\end{array}$ & $\begin{array}{c}\text { Temperatura } \\
\text { Interna Medida } \\
\text { por el Prototipo } \\
\text { (ㄷ) }\end{array}$ & $\begin{array}{c}\text { Error } \\
\text { Porcentual } \\
(\%)\end{array}$ & $\begin{array}{c}\text { Temperatura } \\
\text { Externa Medida } \\
\text { por el } \\
\text { Termametre } \\
\left.\text { Fluke ( }{ }^{\circ} \mathrm{C}\right) \\
\end{array}$ & $\begin{array}{c}\text { Temperatura } \\
\text { Externa Medida } \\
\text { por el Prototipo } \\
\left({ }^{\circ} \mathrm{C}\right)\end{array}$ & $\begin{array}{c}\text { Error } \\
\text { Porcentual } \\
(\%)\end{array}$ \\
\hline 29,1 & 30 & $-3,09$ & 26,6 & 27 & $-1,50$ \\
\hline 36,2 & 37 & $-2,21$ & 26,5 & 27 & $-1,89$ \\
\hline 35,8 & 36 & $-0,56$ & 27 & 27 & 0,00 \\
\hline 33,7 & 33 & 2,08 & 26,8 & 27 & $-0,75$ \\
\hline 42,1 & 42 & 0,24 & 26,2 & 27 & $-3,05$ \\
\hline 31,9 & 32 & $-0,31$ & 26 & 26 & 0,00 \\
\hline 31,3 & 31 & 0,96 & 25,4 & 26 & $-2,36$ \\
\hline 33,5 & 34 & $-1,49$ & 25 & 26 & $-4,00$ \\
\hline 30,1 & 31 & $-2,99$ & 25,2 & 26 & $-3,17$ \\
\hline 34,2 & 35 & $-2,34$ & 25,7 & 26 & $-1,17$ \\
\hline 30,2 & 30 & 0,66 & 26 & 27 & $-3,85$ \\
\hline 30 & 29 & 3,33 & 26,1 & 26 & 0,38 \\
\hline 34,2 & 35 & $-2,34$ & 26,5 & 26 & 1,89 \\
\hline 39,4 & 39 & 1,02 & 25,2 & 26 & $-3,17$ \\
\hline 37,2 & 37 & 0,54 & 26,3 & 27 & $-2,66$ \\
\hline 32,8 & 33 & $-0,61$ & 26,8 & 27 & $-0,75$ \\
\hline 29,2 & 29 & 0,68 & 26,9 & 26 & 3,35 \\
\hline 29,2 & 29 & 0,68 & 26,1 & 25 & 4,21 \\
\hline 33,8 & 34 & $-0,69$ & 26,4 & 25 & 5,30 \\
\hline 38,9 & 39 & $-0,26$ & 26,1 & 25 & 4,21 \\
\hline
\end{tabular}

Tabla 2. Datos obtenidos para la temperatura interna del transformador y del medio ambiente.

La Figura 13 presenta la gráfica de los valores de tensión arrojados por el circuito de acondicionamiento de señal, en función de la temperatura interna del transformador. Nótese el comportamiento lineal del sistema.

Además de las pruebas de temperatura, también se observó detenidamente el comportamiento del canal de transmisión y de los dispositivos de comunicación $R F$, al introducir en la sala de máquinas una serie de elementos que podrían generar algún tipo de interferencia, tales como celulares, radios AM$F M$, radio bases de banda ciudadana y equipos de red inalámbrica. En ningún momento se registró pérdida de datos en el microcontrolador del prototipo receptor, ni pérdidas momentáneas de la comunicación, por lo cual es posible afirmar que el sistema se comporta de manera óptima cuando se trata de establecer una comunicación a cortas distancias, como las existentes entre los transformadores de distribución instalados en los postes y subestaciones, y el personal técnico 
encargado de su instalación, mantenimiento y reparación. También se realizó una prueba de cobertura, detectándose pérdida de la comunicación cuando transmisor y receptor se encontraban separados por una distancia de $50 \mathrm{~m}$, lo cual se identificó al desplazar el receptor a otro salón del laboratorio.

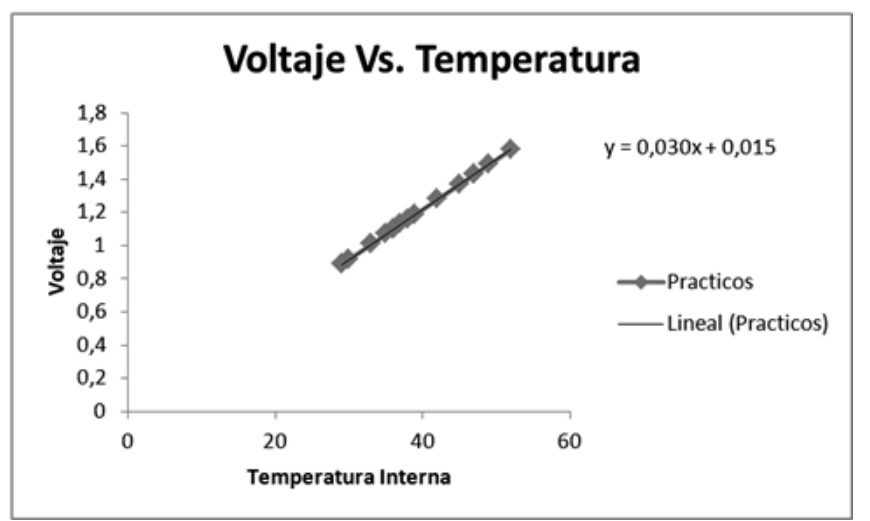

Figura 13. Gráfico de voltaje Vs. temperatura interna del transformador.

\section{CONCLUSIONES}

Uno de los principales factores que se ven involucrados en la vida útil de un transformador es la temperatura. Esto hace que su medición sea de suma importancia, no solamente con el fin de preservar su buen estado, sino también para detectar anomalías, y de esta manera evitar daños futuros. Sin embargo, es muy difícil realizar la medición de la temperatura en los transformadores de manera directa, pues generalmente se encuentran en sitios de difícil acceso, tales como postes de redes de energía o subestaciones eléctricas. Así pues, el desarrollo de un sistema remoto para la medición de esta variable, permite acceder a la información de manera fácil, segura y rápida. Además, en el equipo receptor es posible implementar un sistema de información que almacene el historial de temperaturas, mediante el cual es posible establecer la causa de anomalías presentadas, o rediseñar el sistema eléctrico al originarse sobre carga.

A pesar de que la gran mayoría de soluciones desarrolladas a nivel mundial con el fin de enfrentar el problema de la medición de la temperatura presente en los transformadores de distribución se realiza generando sistemas que ofrecen conectividad a redes de datos eléctricas o de fibra óptica, es difícil implementar este tipo de equipos en Colombia, ya que no se cuenta con una red de datos que interconecte las máquinas de las empresas de energía que se encuentran en las calles. Así pues, la solución propuesta en este artículo se ajusta más a la realidad de la red de distribución eléctrica nacional, al seguir considerando como clave la visita del personal experto al transformador en estudio, pero ofreciendo seguridad, facilidad en la operación, y optimización del tiempo, al eliminar el contacto directo entre la máquina y el personal técnico a la hora de medir los parámetros del transformador.
En cuanto a los resultados obtenidos se refiere, la tabla construida muestra una alta similitud entre los datos arrojados por el prototipo y el equipo patrón, demostrando de esta manera la confiabilidad del sistema. Así pues, el equipo desarrollado presenta como ventaja un conjunto de mediciones de buena precisión, alta seguridad (debido a que ya no es necesaria la interacción directa entre el técnico y la máquina), y la optimización del tiempo de servicio que las empresas de energía invierten en el mantenimiento preventivo de los transformadores.

Si se desea identificar una desventaja del prototipo, se podría pensar en su baja cobertura, pues más allá de $50 \mathrm{~m}$ se pierde la comunicación, aunque esta distancia es más que suficiente para hacer viable la solución propuesta. Aun así, en un posible trabajo futuro se podrían idear sistemas de comunicación de mayor cobertura, de tal manera que los datos sean transmitidos de manera directa a la sala de control de la empresa de energía, eliminando de esta forma la visita del personal técnico a la máquina, por lo menos en cuanto a tareas de monitoreo se refiere.

\section{REFERENCIAS}

[1] S. J. Chapman, Máquinas Eléctricas. México: McGrawHill, 2005, p. 350.

[2] E. Ras Oliva, Transformadores de Potencia, de Medida y de Distribución. Madrid: Editorial Marcombo S.A., 1998, p. 246.

[3] L. Cuéllar, E. Oviedo, "Manejo Eficiente de la Energía Aplicada en Transformadores", Eng. dissertation, Dept. Electrical Eng., Facultad de Ingeniería, Univ. Autónoma de Occidente, Cali, 2007.

[4] E.E. Staff of the M.I.T, Circuitos Magnéticos $y$ Transformadores, 5 ed. Barcelona: Editorial Reverté S.A, 2003, p. 213.

[5] A. Franzén, L. Bertling. (2007). State of the art-life time modeling and management of transformers. KTH Electrical Engineering. $\quad[\mathrm{On} \quad$ Line]. Available: http://www.foxgo.net/uploads/2/1/3/8/2138775/foxgo-

life time_modelling_of transformers.pdf

[6] Treetech, DTM CA-027 Temperature Monitor Datasheet. Disponible en: www.treetech.com.br [consultado el 15 de marzo de 2010].

[7] J. Q. Feng, P. Sun, W. H. Tang, D. P. Buse, Q. H. Wu, Z. Richardson, Z. Fitch, "Implementation of a Power Transformer Temperature Monitoring System", in Proc. 2002 International Conference on Power System Technology, pp. 1980-1983.

[8] S. Pan, P. Zheng, Y. Ren, C. Chen, Z. Shao, "Research on the Remoter Monitoring and Control System of Transformer Winding Temperature Based on Optical Fiber Networked Sensor", in Proc. 2006 6th World Congress on Intelligent Control and Automation, pp. 7627-7631.

[9] D. Dolezilek, B. McDermott, "Remote Data Monitoring and Data Analysis for Substations: A Case Study in 
Implementation”, in Proc. 2005 18th International Conference on Electricity Distribution, pp. 496-500.

[10] W. Chunming,; G. Quiang, "The Transformer Station Remote Monitoring System Based on ARM/GPRS Network", in Proc. 2010 2nd International Workshop on Intelligent Systems and Applications, pp. 1-4.

[11] J. P. Bentley, Sistemas de Medición: Principios y Aplicaciones. México: Compañía Editorial Contienental S.A., 2006, p. 450.

[12] R. A. Pease, A New Celsius Temperature Sensor. National Semiconductor Corp., 1983, p. 65.

[13] A. Sedra, K. C. Smith, Circuitos Microelectrónicos. México: Oxford University Press, 1998, p. 65.

[14] J. A. González Vázquez, Introducción a los Microcontroladores. Madrid: McGraw-Hill, 1992, p. 215. 